\title{
STAT3-induced WNT5A signaling loop in embryonic stem cells, adult normal tissues, chronic persistent inflammation, rheumatoid arthritis and cancer (Review)
}

\author{
MASUKO KATOH ${ }^{1}$ and MASARU KATOH ${ }^{2}$ \\ ${ }^{1}$ M\&M Medical BioInformatics, Hongo 113-0033; ${ }^{2}$ Genetics and Cell Biology Section, \\ National Cancer Center Research Institute, Tokyo 104-0045, Japan
}

Received October 19, 2006; Accepted November 27, 2006

\begin{abstract}
Leukemia inhibitory factor (LIF), oncostatin M, leptin, ciliary neurotrophic factor, cardiotrophin 1 , cardiotrophin-like cytokine factor 1, interleukin 6 (IL6), interleukin 11 and interleukin 27 activate the gp130-JAKSTAT3 signaling cascade. Here, WNT5A was characterized as the evolutionarily conserved target of the STAT3 signaling cascade based on 11-bp-spaced tandem STAT3-binding sites within intron 4 of human, chimpanzee, cow, mouse and rat WNT5A orthologs. Canonical WNT5A signaling through Frizzled and LRP5/LRP6 receptors activates FGF20, WISP1, $M Y C$ and $C C N D 1$ transcription for the maintenance of stem/ progenitor cells, while non-canonical WNT5A signaling through Frizzled and ROR2/PTK7/RYK receptors activates the RHOA, JNK, NLK and NFAT signaling cascades for the control of tissue polarity, cell adhesion or movement. LIFinduced Wnt5a activates canonical Wnt signaling in mouse embryonic stem cells for self-renewal. STAT3-induced Wnt5a activates non-canonical Wnt signaling in rat cardiac myocytes for N-cadherin-dependent aggregation. IL6, secreted from epithelial cells or macrophages, induces WNT5A upregulation in mesenchymal cells. WNT5A then activates canonical WNT signaling in epithelial cells. IL6-induced WNT5A activates canonical WNT signaling for autocrine proliferation of human synovial fibroblasts in rheumatoid arthritis. IL-6 signaling is activated during human chronic atrophic gastritis with Helicobacter pylori infection, and aberrant Stat3 signaling activation gives rise to mouse gastric tumors. WNT5A is frequently upregulated in human primary gastric cancer due to tumor-stromal interaction. WNT5A might be
\end{abstract}

Correspondence to: Dr Masaru Katoh, Genetics and Cell Biology Section, National Cancer Center Research Institute, 5-1-1 Tsukiji, Chuo-ku, Tokyo 104-0045, Japan

E-mail: mkatoh-kkr@umin.ac.jp

Key words: embryonic stem cells, STAT3, WNT5A, Helicobacter pylori, carcinogenesis, bioinformatics, integromics, systems medicine downregulated in advanced cancer with poorer prognosis due to genetic alterations compensating WNT5A signaling. Oncogenic WNT5A activates canonical WNT signaling in cancer stem cells for self-renewal, and non-canonical WNT signaling at the tumor-stromal interface for invasion and metastasis. SNP of genes encoding components of the cytokine-induced WNT5A signaling loop is a predicted risk factor for RA and cancer, especially diffuse-type gastric and pancreatic cancer. Humanized anti-IL6 receptor antibody and WNT5A mimetic small-molecule antagonist could be applied to personalized medicine for RA and cancer driven by the IL6-induced WNT5A signaling loop.

\section{Contents}

1. WNT5A signaling overview

2. STAT3-dependent WNT5A upregulation

3. LIF-induced WNT5A signaling loop for embryonic stem cells

4. Leptin-induced WNT5A signaling loop for cardiac myocytes

5. IL6-induced WNT5A signaling loop in rheumatoid arthritis

6. IL6-induced WNT5A signaling loop in chronic inflammation and cancer

7. Perspectives

\section{WNT5A signaling overview}

WNT5A is a secreted glycoprotein, belonging to the WNT family (1-4). WNT5A signaling through Frizzled (FZD) family receptor and LRP5/LRP6 co-receptor is transduced to the canonical WNT signaling cascade for transcriptional activation of target genes based on the nuclear complex, consisting of TCF/LEF, B-catenin, BCL9/BCL9L and PYGO1/PYGO2 (5-9). The FGF20, JAG1, DKK1 and WISP1 genes are primary transcriptional targets of the canonical WNT signaling pathway (10-14). On the other hand, WNT5A signaling through FZD family receptor and ROR2/PTK7/ RYK co-receptor is transduced to a variety of non-canonical WNT signaling cascades, such as the Dishevelled-dependent RHOA/RHOU/RAC/CDC42, the Dishevelled-dependent 


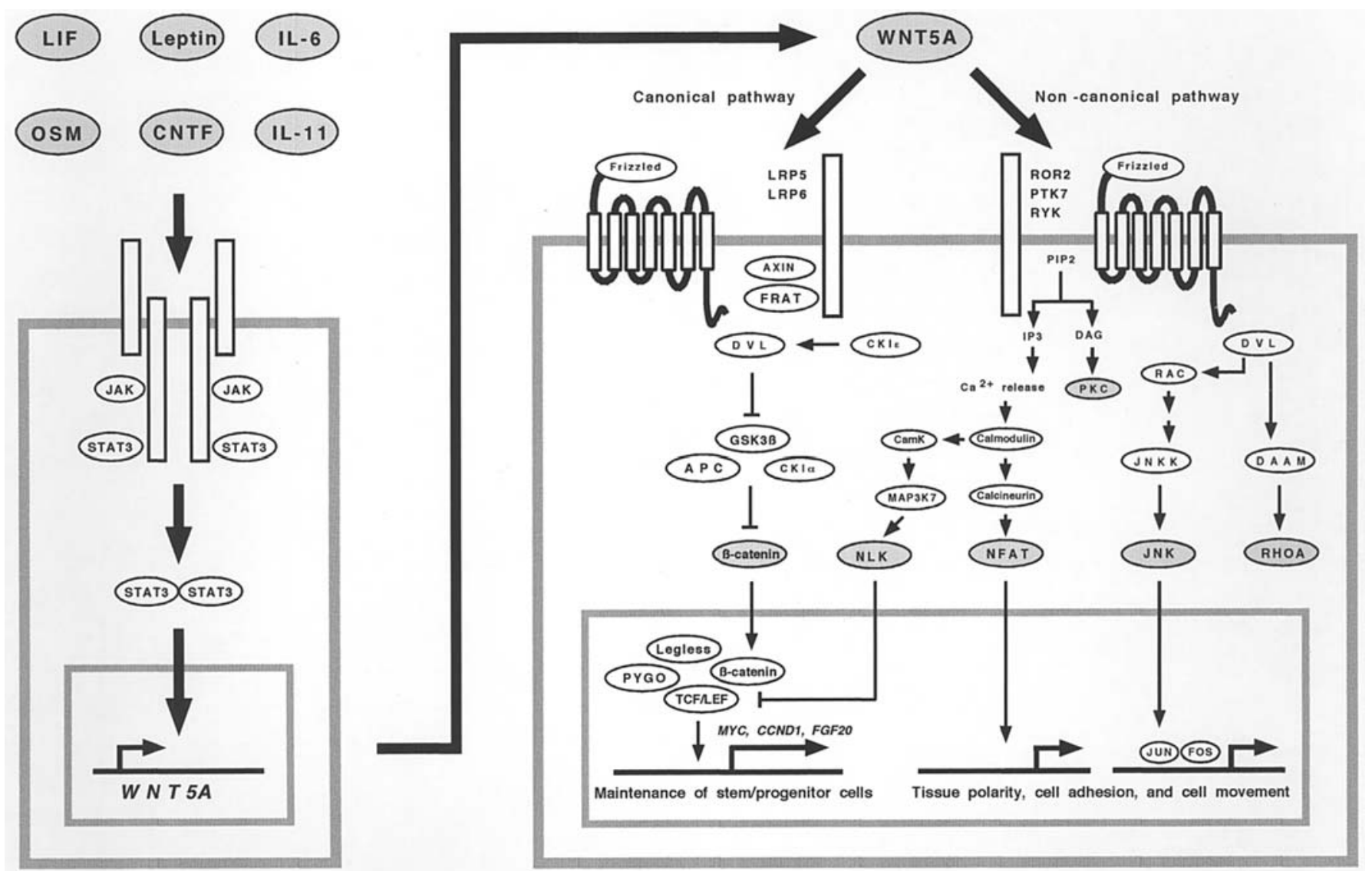

Figure 1. Overview of the cytokine-induced WNT5A signaling loop. IL6 family members, such as LIF, OSM, Leptin, CNTF, IL6 and IL11, activate the gp130-JAK-STAT3 signaling cascade to upregulate WNT5A transcription. WNT5A signal is transduced to canonical and non-canonical WNT signaling cascades in a context-dependent manner. Canonical WNT5A signaling through Frizzled receptor and LRP5/LRP6 co-receptor is transduced to the $\beta$-catenin signaling cascade for the maintenance of stem/progenitor cells. Non-canonical WNT5A signaling through Frizzled receptor and ROR2/PTK7/RYK coreceptor is transduced to RHOA, JNK, NLK and NFAT signaling cascades for the control of tissue polarity, cell adhesion, or cell movement.

JNK, the $\mathrm{Ca}^{2+}$-dependent NLK and the $\mathrm{Ca}^{2+}$-dependent NFAT signaling cascades (15-21). WNT5A signals are transduced to the canonical WNT signaling cascade for the maintenance of stem and progenitor cells, and to the noncanonical WNT signaling cascades for the control of tissue polarity, cell adhesion, and cell movement (Fig. 1).

A combination of Wnt5a and FZD5 activates the canonical WNT signaling pathway, while Wnt5a itself inhibits the canonical WNT signaling pathway through NLKmediated phosphorylation of TCF/LEF family transcription factors. WNT5A signals are context-dependently transduced to the canonical WNT signaling cascade and the noncanonical WNT signaling cascades based on the expression profile of FZD family receptors, co-receptors, and the activity of cytoplasmic WNT signaling regulators (Fig. 1).

\section{STAT3-induced WNT5A upregulation}

LIF (leukemia inhibitory factor) and CTF1 (cardiotrophin 1) are reported to induce Wnt5a upregulation through the Stat3 signaling cascade in rat cardiac myocytes (22); however, the transcriptional mechanism of Stat3-induced Wnt5a upregulation remained unclear.

Here, we searched for evolutionarily conserved STAT3binding sites within WNT5A orthologs to elucidate the mechanism for STAT3-dependent WNT5A upregulation. We previously identified and characterized the rat Wnt5a gene for comparative genomic analyses, and reported that the promoter and five exonic regions are well conserved between the human WNT5A and rat Wnt5a genes (23). The single STAT3-binding site within the human WNT5A 5'-promoter was not evolutionarily conserved, and the STAT3-binding site was not identified within the human WNT5A exonic regions (data not shown). Next, the STAT3-binding sites within the intronic regions were searched for. Tandem STAT3binding sites with 11-bp spacing were successfully identified within the conserved region in intron 4 (Fig. 2). The tandem STAT-binding sites within intron 4 were conserved among human, chimpanzee, cow, mouse, and rat WNT5A orthologs. Based on these facts, it was concluded that mammalian WNT5A orthologs were STAT3-target genes (Fig. 1).

The IL6 cytokine family consists of LIF, OSM (oncostatin $\mathrm{M})$, LEP (leptin), CNTF (ciliary neurotrophic factor), CTF1, CLCF1 (cardiotrophin-like cytokine factor 1), IL6 (interleukin 6), IL11 (interleukin 11) and IL27 (interleukin 27) (24-32). Signals of the IL6 family cytokines are transduced through ligand-specific receptor and IL6ST (gp130) transducer to JAK kinase for IL6ST phosphorylation, which results in STAT3 signaling activation $(33,34)$. The IL6 family cytokines, such as LIF, OSM, LEP, CNTF, IL6 and IL11, 


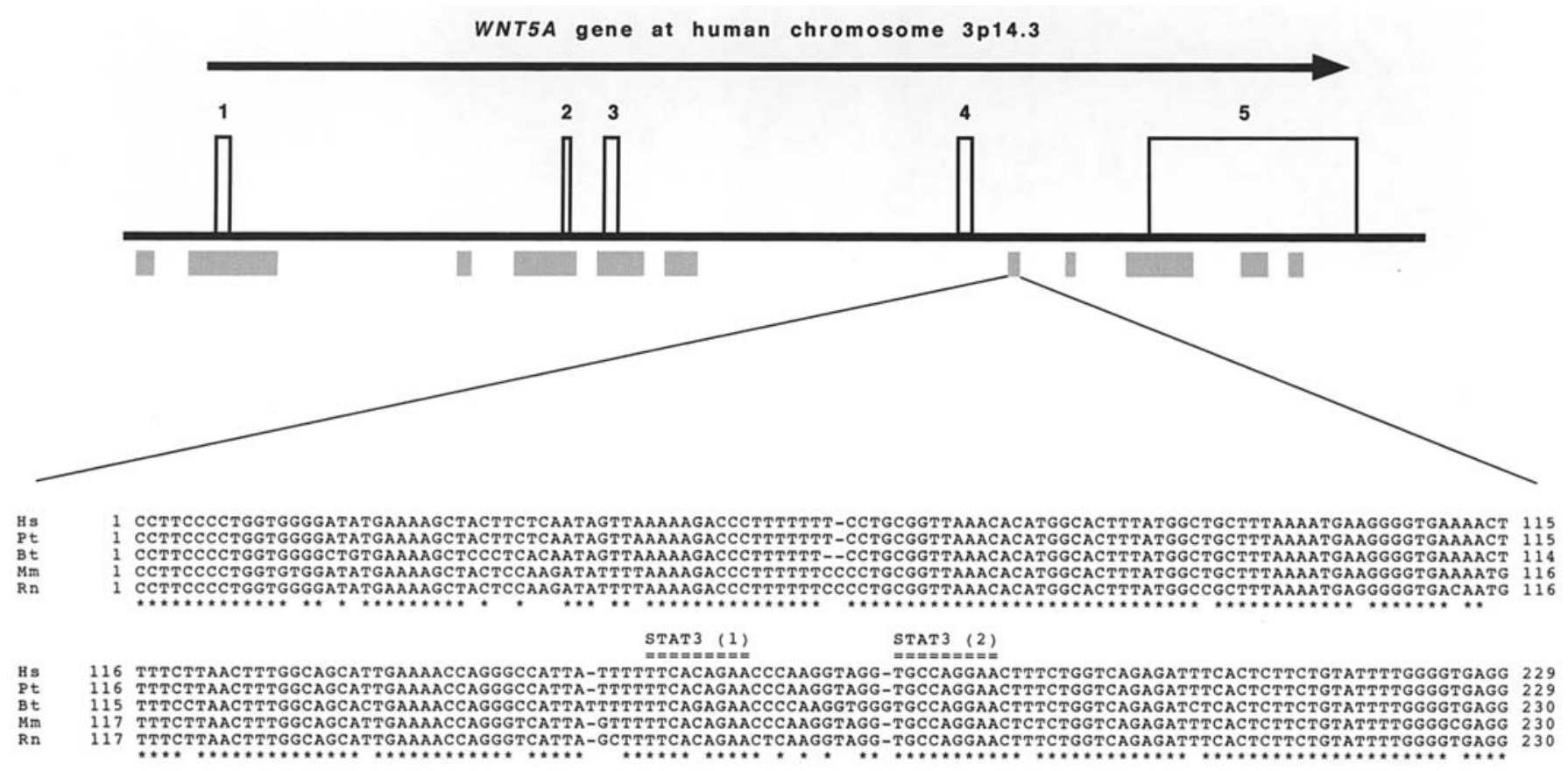

Figure 2. STAT3-binding sites within WNT5A orthologs. The human WNT5A gene consists of five exons. Regions conserved between the human WNT5A and rat Wnt5a genes are shown by gray bars. Tandem STAT3-binding sites with 11-bp spacing within intron 4 of the human WNT5A gene are conserved in chimpanzee, cow, mouse and rat WNT5A orthologs. The WNT5A gene is the evolutionarily conserved target of the STAT3 signaling cascade.

Cytokine secreting cell
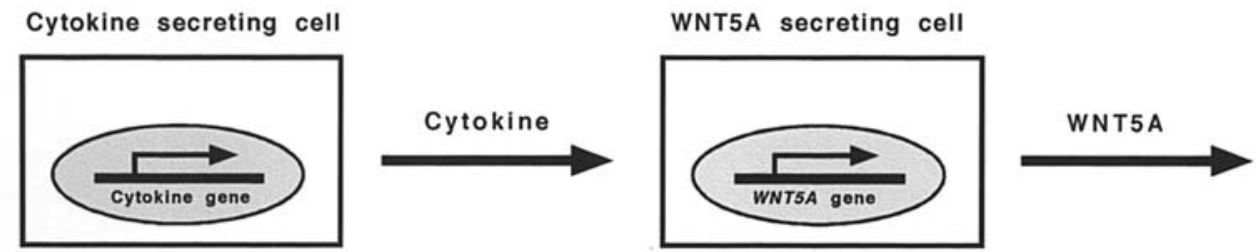

WNT5A target cell

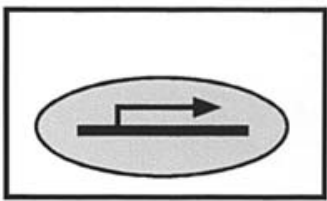

\begin{tabular}{|c|c|c|c|c|}
\hline Cytokine & $\begin{array}{l}\text { Cytokine target cells } \\
\text { (WNT5A secreting cells) }\end{array}$ & WNT5A target cells & WNT5A signaling & Effects of WNT5A \\
\hline LIF & Mouse embryonic stem cells & Mouse embryonic stem cells & Canonical & Maintenance of self-renewal \\
\hline $\begin{array}{l}\text { LIF } \\
\text { CTF1 }\end{array}$ & Cardiac myocytes & Cardiac myocytes & Non-canonical & Cardiac myocyte aggregation \\
\hline IL6 & $\begin{array}{l}\text { Rheumatoid synovial fibroblasts } \\
\text { Tumor-infliltrating macrophages } \\
\text { Invasion-front fibroblasts }\end{array}$ & $\begin{array}{l}\text { Rheumatold synivial fibroblasts } \\
\text { Tumor cells } \\
\text { Tumor cells }\end{array}$ & $\begin{array}{l}\text { Canonical } \\
\text { Canonical } \\
\text { Non-canonical }\end{array}$ & $\begin{array}{l}\text { Synovial fibroblast hypertrophy } \\
\text { Maintenance of cancer stem cells } \\
\text { Invasion and metastasis }\end{array}$ \\
\hline
\end{tabular}

Figure 3. The biological roles of the cytokine-induced WNT5A signaling loop. LIF-induced WNT5A activates canonical WNT signaling for self-renewal in mouse embryonic stem cells. Cytokine-induced WNT5A activates non-canonical WNT signaling to prevent cardiac myocyte hypertrophy. IL6-induced WNT5A activates canonical WNT signaling for synovial fibroblast hypertrophy in rheumatoid arthritis. IL6-induced WNT5A activates canonical WNT signaling in cancer stem cells for self-renewal, and non-canonical WNT signaling at the tumor-stromal interface for invasion and metastasis.

bind to cell surface receptors for IL6ST-JAK-STAT3induced WNT5A upregulation. WNT5A then binds to Frizzled receptor and co-receptor for WNT signaling activation in a context-dependent manner (Fig. 1). The WNT5A signaling loop is defined as the STAT3-induced WNT5A upregulation (Fig. 3). The biological roles of the WNT5A signaling loop in a variety of physiological or pathological processes will be described in the following sections.

\section{LIF-induced WNT5A signaling loop for embryonic stem cells}

LIF-induced Stat3 signaling activation is sufficient for the maintenance of pluripotency or self-renewal in mouse embryonic stem cells (35), and canonical Wnt signaling is necessary for the self-renewal of mouse embryonic stem cells (36). Wnt5a, secreted from feeder cells, activates the canonical Wnt signaling pathway in mouse embryonic stem cells (37). 
The $M y c$ gene is the common transcriptional target of the canonical WNT and LIF-Stat3 signaling cascades, and Myc protein is further stabilized by the canonical Wnt signaling cascade (38). Because Wnt5a and LIF synergistically enhance the self-renewal potential of mouse embryonic stem cells, feeder cells secreting larger amounts of Wnt5a more effectively maintain undifferentiated mouse embryonic stem cells. The LIF-induced Wnt5a signaling loop plays a key role in the self-renewal of mouse embryonic stem cells.

WNT signaling activation is reported to be sufficient for the self-renewal of human embryonic stem cells (36); however, LIF-STAT3 or WNT signaling alone is insufficient for the self-renewal of human embryonic stem cells $(39,40)$. NODAL signaling activation is essential for the self-renewal of human embryonic stem cells, and WNT signaling cooperates with NODAL signaling (40). NODAL antagonist CER1 (Cerberus 1) is the common target of the NODAL and WNT signaling pathways in human embryonic stem cells, but not in mouse embryonic stem cells (41). LIF, IL6ST, JAK1, STAT3, and WNT5A mRNAs are expressed in embryoid body derived from human embryonic stem cells rather than undifferentiated human embryonic stem cells (42). The LIFinduced WNT5A signaling loop is activated during the differentiation process of human embryonic stem cells for embryoid body formation.

\section{Cytokine-induced WNT5A signaling loop for cardiac myocytes}

LIF and CTF1 activate the IL6ST-JAK-STAT3 signaling cascade for WNT5A upregulation in cardiac myocytes (Fig. 1). WNT5A then activates non-canonical WNT signaling to induce protein stabilization of N-cadherin for cardiac myocyte aggregation (22).

Leptin is mainly secreted from mature adipocytes for endocrine signal transduction to a variety of tissues, such as hypothalamus, skeletal muscle, liver, pancreas, and heart $(26,43,44)$. Leptin signaling to the hypothalamus is involved in the regulation of food intake, and this signaling to skeletal muscle, the liver and the pancreas is involved in lipid and glucose metabolism. The leptin receptor is also expressed in the heart, especially in cardiac myocytes. Leptin elevation in patients with metabolic syndrome leads to abrogation of leptin signaling (leptin resistance) through the induction of a secreted leptin antagonist CRP (45). Leptin deficiency as well as leptin resistance lead to left ventricular hypertrophy and congestive heart failure. CNTF rescues left ventricular hypertrophy associated with leptin resistance through the CNTF-receptor-mediated STAT3-signaling activation (46). Together, these facts indicate that the cytokine-induced WNT5A signaling loop in cardiac myocytes is implicated in the prevention of left ventricular hypertrophy.

\section{IL6-induced WNT5A signaling loop in rheumatoid arthritis}

Rheumatoid arthritis, triggered by joint vasculitis, leads to the infiltration of inflammatory cells into the synovium. Inflammatory leukoctyes promote the proliferation of synovial fibroblasts in patients with rheumatoid arthritis. Activated synovial fibroblasts then induce the differentiation of synovial macrophages into osteoclasts for cartilage destruction.

IL6 in the synovial fluid is significantly elevated in patients with rheumatoid arthritis (47), and synovial IL6 activates the IL6ST-JAK-STAT3 signaling cascade in synovial fibroblasts (48). WNT5A and FZD5 are upregulated in synovial fibroblasts of patients with rheumatoid arthritis (49). Downregulation of WNT5A expression in synovial fibroblasts by using WNT5A anti-sense construct as well as by the inhibition of FZD5 signaling using anti-FZD5 antibody inhibits rheumatoid synovial fibroblast activation (50). Because WNT5A activates the canonical WNT signaling cascade through FZD5 and LRP5/LRP6 (5), the IL6-induced WNT5A signaling loop is implicated in synovial fibroblast hypertrophy in rheumatoid arthritis (Fig. 1).

\section{IL6-induced WNT5A signaling loop in chronic inflammation and carcinogenesis}

Helicobacter pylori, Gram-negative bacteria colonized to gastric mucosa, is a causative agent for peptic ulcer diseases, chronic gastritis, and gastric cancer (51-53). Helicobacter pylori induces IL6 expression in infiltrating macrophages and gastric epithelial cells $(54,55)$. IL6 activates the IL6ST-JAKSTAT3 signaling cascade in mesenchymal or stromal cells in the stomach during chronic Helicobacter pylori infection, which leads to WNT5A upregulation (Fig. 1). WNT5A then activates the canonical WNT signaling cascade in epithelial progenitor cells for the maintenance of the stem/progenitor cell population, and also the non-canonical WNT signaling cascades in differentiating cells to promote motility for mucosal repair. On the other hand, gp130 ( $\triangle$ STAT) mice with STAT3 signaling abrogation show impaired wound healing in colonic mucosa (56). Because Wnt5a is expressed in the mesenchymal cells around the crypt base of mouse intestinal epithelium for the activation of Wnt signaling in progenitor cells (57), STAT3 signaling abrogation results in impaired wound healing. The cytokine-induced WNT5A signaling loop is activated for the mucosal restitution during chronic persistent inflammation, which might explain the link between chronic persistent inflammation and carcinogenesis.

We reported WNT5A upregulation in five of eight cases of primary gastric cancer by using matched tumor/normal expression array analysis, and in seven of ten other cases of primary gastric cancer by using cDNA-PCR (3). Compared to frequent WNT5A upregulation in primary gastric cancer, expression levels of WNT5A in seven gastric cancer cell lines were significantly lower than that in the normal stomach, indicating that frequent WNT5A upregulation in primary gastric cancer is due to cancer-stromal interaction (3). Gp130 (757F) mice with aberrant IL6ST-JAK-STAT3 signaling activation developed gastric adenomas by three months of age (56). Together, these facts indicate that the aberrant activation of the STAT3-dependent WNT5A signaling loop is oncogenic in gastric mucosa.

We also reported upregulation of WNT5A in five of 18 cases of primary colorectal tumors, in two of seven cases of primary uterine tumors by using matched tumor/normal expression array analysis (3), and also frequent expression of WNT5A in cervical and embryonal cancer (58). Iozzo RV et al 
reported upregulation of WNT5A in lung cancer, breast cancer, prostate cancer, and melanoma (2). Weeraratna AT et al reported that WNT5A signaling affects motility and invasion of metastatic melanoma (59). WNT5A upregulation leads to a more malignant phenotype in a variety of human cancers through WNT signaling activation.

WNT5A is upregulated in early-stage primary tumors due to tumor-stromal interaction. On the other hand, WNT5A may be downregulated in late-stage primary tumors due to the absolute abundance of tumor cells over contaminating stromal cells, and/or due to genetic alterations compensating WNT5A signaling from stromal cells. In addition, stromal WNT5A at the invasion front activates the non-canonical WNT signaling pathway to promote invasion and metastasis of cancer cells. Therefore, even if the prognosis of patients with reduced WNT5A expression is poor, WNT5A is oncogenic during carcinogenesis.

\section{Perspectives}

Cytokine-induced WNT5A signaling to the canonical WNT pathway in pathological conditions induces rheumatoid arthritis and a variety of cancers associated with chronic inflammation, while cytokine-induced WNT5A signaling to the non-canonical WNT pathway in a physiological condition maintains tissue homeostasis. Because a pathological condition associated with the cytokine-induced canonical WNT5A signaling activation is characterized by the aberrant activation and proliferation of mesenchymal cells, cytokineinduced canonical WNT5A signaling might also be implicated in tissue fibrosis or glial scars associated with STAT3 signaling activation. Therefore, the role of the cytokineinduced WNT5A signaling loop during liver cirrhosis, pulmonary fibrosis, and spinal cord injury should be further investigated.

Medical genome science based on a high-throughput experimental system and high-speed computation is the driving force for medical transformation (59). Exploration of single nucleotide polymorphism (SNP) associated with disease is facilitated in the post-genome era. Systemic SNP analyses could elucidate the link between the cytokinedependent WNT5A signaling loop and various pathological conditions associated with the aberrant activation and proliferation of mesenchymal cells mentioned above. SNP of genes encoding cytokine-dependent WNT5A signaling components are predicted risk factors for rheumatoid arthritis and a variety of cancers, especially diffuse type gastric and pancreatic cancer.

Humanized or human monoclonal antibody as well as small-molecule inhibitors targeted to the cytokine-induced WNT5A signaling loop are promising drugs in the postgenome era (60). Safholm A et al developed formylated hexapeptide WNT5A mimetic compound to block the noncanonical WNT signaling pathway (61); however, a smallmolecule WNT5A antagonist to inhibit the canonical WNT5A signaling pathway must be developed for the treatment of rheumatoid arthritis and cancer. Humanized anti-IL6 receptor antibody developed by Dr Kishimoto's group is a promising drug for rheumatoid arthritis (62). Because the IL6-induced WNT5A signaling loop activates the canonical WNT signaling pathway in pathological conditions, humanized anti-IL6 receptor antibody is predicted to be effective not only for rheumatoid arthritis, but also for human cancer with IL6-dependent WNT5A upregulation.

\section{References}

1. Clark CC, Cohen I, Eichstetter I, et al: Molecular cloning of the human proto-oncogene Wnt-5 A and mapping of the gene (WNT5A) to chromosome 3p14-p21. Genomics 18: 249-260, 1993.

2. Iozzo RV, Eichstetter I and Danielson KG: Aberrant expression of the growth factor Wnt-5A in human malignancy. Cancer Res 55: 3495-3499, 1995.

3. Saitoh T and Katoh M: Frequent up-regulation of WNT5A mRNA in primary gastric cancer. Int J Mol Med 9: 515-519, 2002.

4. Katoh M: $W N T$ and $F G F$ gene clusters. Int $\mathrm{J}$ Oncol 21: 1269-1273, 2002.

5. He X, Saint-Jeannet JP, Wang Y, et al: A member of the Frizzled protein family mediating axis induction by Wnt-5A. Science 275: 1652-1654, 1997.

6. Pinson KI, Brennan J, Monkley S, et al: An LDL-receptorrelated protein mediates Wnt signalling in mice. Nature 407 535-538, 2000.

7. Kramps T, Peter O, Brunner E, et al: Wnt/wingless signaling requires BCL9/legless-mediated recruitment of pygopus to the nuclear B-catenin-TCF complex. Cell 109: 47-60, 2002.

8. Katoh $\mathrm{M}$ and Katoh M: Identification and characterization of human $B C L 9 L$ gene and mouse Bcl9l gene in silico. Int J Mol Med 12: 643-649, 2003

9. Katoh M and Katoh M: Cross-talk of WNT and FGF signaling pathways at GSK3ß to regulate B-catenin and SNAIL signaling cascades. Cancer Biol Ther 5: 1059-1064, 2006.

10. Chamorro MN, Schwartz DR, Vonica A, et al: FGF20 and $D K K 1$ are transcriptional targets of $B$-catenin and FGF20 is implicated in cancer and development. EMBO J 24: 73-84, 2005.

11. Katoh $\mathrm{M}$ and Katoh M: Comparative genomics on FGF2O orthologs. Oncol Rep 14: 287-290, 2005.

12. Katoh M and Katoh M: Notch ligand, JAG1, is evolutionarily conserved target of canonical WNT signaling pathway in progenitor cells. Int J Mol Med 17: 681-685, 2006.

13. Katoh Y and Katoh M: Comparative genomics on DKK1 orthologs. Int J Oncol 27: 275-279, 2005.

14. Pennica D, Swanson TA, Welsh JW, et al: WISP genes are members of the connective tissue growth factor family that are up-regulated in Wntl-transformed cells and aberrantly expressed in human colon tumors. Proc Natl Acad Sci USA 95: $14717-14722,1998$

15. Yamaguchi TP, Bradley A, McMahon AP, et al: A Wnt5a pathway underlies outgrowth of multiple structures in the vertebrate embryo. Development 126: 1211-1223, 1999.

16. Oishi I, Suzuki H, Onishi N, et al: The receptor tyrosine kinase Ror2 is involved in non-canonical Wnt5a/JNK signalling pathway. Genes Cells 8: 645-654, 2003.

17. Lu X, Borchers AG, Jolicoeur C, et al: PTK7/CCK-4 is a novel regulator of planar cell polarity in vertebrates. Nature 430: 93-98, 2004.

18. Lu W, Yamamoto V, Ortega B, et al: Mammalian Ryk is a Wnt coreceptor required for stimulation of neurite outgrowth. Cell 119: 97-108, 2004.

19. Boutros M, Paricio N, Strutt DI, et al: Dishevelled activates JNK and discriminates between JNK pathways in planar polarity and wingless signaling. Cell 94: 109-118, 1998.

20. Dejmek J, Safholm A, Kamp Nielsen C, et al: Wnt-5a/Ca ${ }^{2+}$ induced NFAT activity is counteracted by Wnt-5a/Yes-Cdc42casein kinase Ia signaling in human mammary epithelial cells. Mol Cell Biol 26: 6024-6036, 2006.

21. Katoh M: WNT/PCP signaling pathway and human cancer. Oncol Rep 14: 1583-1588, 2005.

22. Fujio Y, Matsuda T, Ohshima Y, et al: Signals through gp130 upregulate Wnt5a and contribute to cell adhesion in cardiac myocytes. FEBS Lett 573: 202-206, 2004

23. Katoh $\mathrm{M}$ and Katoh M: Comparative genomics on WNT5A and $W N T 5 B$ genes. Int J Mol Med 15: 749-753, 2005.

24. Gough NM, Gearing DP, King JA, et al: Molecular cloning and expression of the human homologue of the murine gene encoding myeloid leukemia-inhibitory factor. Proc Natl Acad Sci USA 85: 2623-2627, 1988 
25. Zarling JM, Shoyab M, Marquardt H, et al: Oncostatin M: a growth regulator produced by differentiated histiocytic lymphoma cells. Proc Natl Acad Sci USA 83: 9739-9743, 1986.

26. Zhang Y, Proenca R, Maffei M, et al: Positional cloning of the mouse obese gene and its human homologue. Nature 372: 425-432, 1994.

27. Lam A, Fuller F, Miller J, et al: Sequence and structural organization of the human gene encoding ciliary neurotrophic factor. Gene 102: 271-276, 1991.

28. Pennica D, King KL, Shaw KJ, et al: Expression cloning of cardiotrophin 1, a cytokine that induces cardiac myocyte hypertrophy. Proc Natl Acad Sci USA 92: 1142-1146, 1995.

29. Senaldi G, Varnum BC, Sarmiento U, et al: Novel neurotrophin-1/B cell-stimulating factor-3: a cytokine of the IL-6 family. Proc Natl Acad Sci USA 96: 11458-11463, 1999.

30. Hirano T, Yasukawa K, Harada H, et al: Complementary DNA for a novel human interleukin (BSF-2) that induces B lymphocytes to produce immunoglobulin. Nature 324: 73-76, 1986.

31. Paul SR, Bennett F, Calvetti JA, et al: Molecular cloning of a cDNA encoding interleukin 11 , a stromal cell-derived lymphopoietic and hematopoietic cytokine. Proc Natl Acad Sci USA 87: 7512-7516, 1990.

32. Pflanz S, Hibbert L, Mattson J, et al: WSX-1 and glycoprotein 130 constitute a signal-transducing receptor for IL-27. J Immunol 172: 2225-2231, 2004.

33. Hibi M, Murakami M, Saito M, et al: Molecular cloning and expression of an IL-6 signal transducer, gp130. Cell 63: 1149-1157, 1990.

34. Heinrich PC, Behrmann I, Muller-Newen G, et al: Interleukin6-type cytokine signaling through the gp130/JAK/STAT pathway. Biochem J 334: 297-314, 1998.

35. Chambers I and Smith A: Self-renewal of teratocarcinoma and embryonic stem cells. Oncogene 23: 7150-7160, 2004.

36. Sato N, Meijer L, Skaltsounis L, et al: Maintenance of pluripotency in human and mouse embryonic stem cells through activation of Wnt signaling by a pharmacological GSK3specific inhibitor. Nat Med 10: 55-63, 2004.

37. Hao J, Li TG, Qi X, et al: WNT/B-catenin pathway up-regulates Stat 3 and converges on LIF to prevent differentiation of mouse embryonic stem cells. Dev Biol 290: 81-91, 2006.

38. Cartwright P, McLean C, Sheppard A, et al: LIF/STAT3 controls ES cell self-renewal and pluripotency by a Mycdependent mechanism. Development 132: 885-896, 2005.

39. Humphrey RK, Beattie GM, Lopez AD, et al: Maintenance of pluripotency in human embryonic stem cells is STAT3 independent. Stem Cells 22: 522-530, 2004.

40. Vallier L, Alexander M and Pedersen RA: Activin/Nodal and FGF pathways cooperate to maintain pluripotency of human embryonic stem cells. J Cell Sci 118: 4495-4509, 2005.

41. Katoh M and Katoh M: CER1 is a common target of WNT and NODAL signaling pathways in human embryonic stem cells. Int J Mol Med 17: 795-799, 2006.

42. Brandenberger R, Wei H, Zhang S, et al: Transcriptome characterization elucidates signaling networks that control human ES cell growth and differentiation. Nat Biotech 22: 707-716, 2004.

43. Tartaglia LA, Dembski M, Weng X, et al: Identification and expression cloning of a leptin receptor, OB-R. Cell 83: 1263-1271, 1995.
44. Raguso C and McCullough AJ: Leptin and the gastrointestinal tract. Curr Opin Gastroenterol 16: 160-165, 2000.

45. Chen K, Li F, Li J, et al: Induction of leptin resistance through direct interaction of C-reactive protein with leptin. Nat Med 12: 425-432, 2006.

46. Raju SVY, Zheng M, Schuleri KH, et al: Activation of the cardiac ciliary neurotrophic factor receptor reverses left ventricular hypertrophy in leptin-deficient and leptin-resistant obesity. Proc Natl Acad Sci USA 103: 4222-4227, 2006.

47. Swaak AJ, van Rooyen A, Nieuwenhuis E, et al: Interleukin-6 (IL-6) in synovial fluid and serum of patients with rheumatic diseases. Scand J Rheumatol 17: 469-474, 1988.

48. Krause A, Scaletta N, Ji JD, et al: Rheumatoid arthritis synoviocyte survival is dependent on Stat3. J Immunol 169: 6610-6616, 2002.

49. Sen M, Lauterbach K, El-Gabalawy H, et al: Expression and function of wingless and frizzled homologs in rheumatoid arthritis. Proc Natl Acad Sci USA 97: 2791-2796, 2000.

50. Sen M, Chamorro M, Reifert J, et al: Blockade of Wnt-5A/ frizzled 5 signaling inhibits rheumatoid synoviocyte activation. Arthritis Rheum 44: 772-781, 2001.

51. Peek RM Jr and Crabtree JE: Helicobacter pylori infection and gastric neoplasia. J Pathol 208: 233-248, 2006.

52. Katoh $\mathrm{Y}$ and Katoh M: Hedgehog signaling in gastric cancer. Cancer Biol Ther 4: 1050-1054, 2005.

53. Katoh $\mathrm{M}$ and Katoh M: FGF signaling network in the gastrointestinal tract. Int J Oncol 29: 163-168, 2006.

54. Odenbreit S, Linder S, Gebert-Vogl B, et al: Interleukin-6 induction by Helicobacter pylori in human macrophages is dependent on phagocytosis. Helicobacter 11: 196-207, 2006.

55. Lu H, Wu JY, Kudo T, et al: Regulation of interleukin-6 promoter activation in gastric epithelial cells infected with Helicobacter pylori. Mol Biol Cell 16: 4954-4966, 2005.

56. Tebbutt NC, Giraud AS, Inglese M, et al: Reciprocal regulation of gastrointestinal homeostasis by SHP2 and STAT-mediated trefoil gene activation in gp130 mutant mice. Nat Med 8: 1089-1097, 2002.

57. Gregorieff A, Pinto D, Begthel H, et al: Expression pattern of WNT signaling components in the adult intestine. Gastroenterology 129: 626-638, 2005 .

58. Saitoh T and Katoh M: Expression and regulation of WNT5A and $W N T 5 B$ in human cancer: Up-regulation of $W N T 5 A$ by TNF $\alpha$ in MKN45 cells and down-regulation of WNT5B by B-estradiol in MCF-7 cells. Int J Mol Med 10: 345-349, 2002.

59. Weeraratna AT, Jiang Y, Hostetter G, et al: Wnt5a signaling directly affects cell motility and invasion of metastatic melanoma. Cancer Cell 1: 279-288, 2002.

60. Katoh M and Katoh M: Bioinformatics for cancer management in the post-genome era. Technol Cancer Res Treat 5: 169-176, 2006.

61. Safholm A, Leandersson K, Dejmek J, et al: A formylated hexapeptide ligand mimics the ability of Wnt-5a to impair migration of human breast epithelial cells. J Biol Chem 281: 2740-2749, 2006.

62. Kishimoto N, Yoshizaki K, Miyasaka N, et al: Treatment of rheumatoid arthritis with humanized anti-interleukin-6 receptor antibody: a multicenter, double-blind, placebo-controlled trial. Arthritis Rheum 50: 1761-1769, 2004. 